\title{
ESTILOS DE APRENDIZAJES Y ENFOQUES DE ENSEÑANZA, CARRERA DE INGENIERÍA AGROFORESTAL
}

Ariel Domingo Chavarría Vigil[ ${ }^{[1]}$ Sergio Genaro Rodríguez Ruíz ${ }^{[2]}$

\section{Resumen}

Se abordaron los estilos de aprendizajes en discentes y enfoques de enseñanza docente en la carrera de Ingeniería Agroforestal Universidad de las Regiones Autónomas de la Costa Caribe Nicaragüense -RAAN-, Recinto Universitario Las Minas, 2008.

La metodología que sustentó esta investigación es carácter cualitativo con un enfoque etnometodológico, las entrevistas individuales y la observación, se recopiló, procesó y analizó los datos que fueron proporcionados por los sujetos de estudio.

Los resultados revelaron que los estilos en los procesos de enseñanza corresponden a estilos divergentes y se desarrollan en torno a dos enfoques a saber: el transmisivo y el constructivista. El primero predomina en la mayoría de los docentes y el último en una minoría.

La relación entre los enfoques de enseñanza con los estilos de aprendizaje se da en un nivel no significativo con respecto a las características de los estilos identificados. Destacando que ninguno de los docentes entrevistados conoce el estilo de aprendizaje de sus discentes.

Este esfuerzo permitirá articular propuestas que den pautas sobre la relación que debe darse entre docente y discente en el éxito de la enseñanza. Resultados que servirán de base para implementar estrategias didácticas.

PALABRAS CLAVES: Estilos de aprendizajes, enfoque de enseñanza, educación, estrategias de enseñanza y Pedagogía.

\section{Introducción}

Los estilos de aprendizajes constituyen un aspecto central del proceso educativo, tanto así que los especialistas en esta temática los consideran un indicador efectivo en los sistemas educativos y en particular en los procesos de enseñanza - aprendizaje que se practican en las aulas de clase. Estos son categorías objeto de estudio en la Educación

[1] MSc. en Docencia Universitaria. Profesor de la URACCAN, Recinto Universitario Las Minas.

[2] MSc. en Docencia Universitaria. Coordinador de Cooperación Externa Recinto Universitario Las Minas. 
Superior y básicamente en las carreras de ingeniería en Europa y Estados Unidos de Norteamérica, por su importancia en el desempeño académico, la satisfacción y eficiencia de los estudiantes.

En países latinoamericanos como Chile, Brasil y Argentina, existen pocos estudios publicados al respecto. No obstante, los estudios existentes señalan la importancia que los estilos de aprendizajes tienen en la manera habitual en que percibimos y procesamos la información durante el aprendizaje, lo que evidencia en gran medida la diversidad que los mismos significan en términos de procesos tanto de enseñanza como de aprendizaje de los discentes.

En consiguiente, se valora los estilos de aprendizaje en la carrera de Ingeniería Agroforestal, asimismo los enfoques de enseñanza docente, con el objetivo de identificarlos y enfatizar en relación con los enfoques en los procesos significativos en el aprendizaje y las sugerencias para su implementación. Los estilos de aprendizajes constituyen categorías relevantes para el desempeño y desarrollo académico de los discentes de agroforestería, se dispone por lo tanto, con una base racional para optimizar la formación profesional.

El espíritu que orientó esta investigación es la acción constructivista en pro de contribuir a revisar la pertinencia de las prácticas de enseñanza. El mismo permitió detectar los principales elementos que inciden de forma positiva y negativa en los estilos de aprendizajes; encontrando diversos saberes, habilidades y actitudes en el marco del proceso enseñanza significativa, dando pautas para fortalecer características personales menos desarrolladas que son necesarias para el éxito profesional.

\section{Revisión de literatura}

Según Salas, R. (1995), referido por Lobo \& Jiménez (2002), el concepto de estilos de aprendizaje se fundamenta en el hecho de las diferencias y se manifiesta en rasgos tales como la edad, experiencia, nivel de conocimiento o intereses, y las características psíquicas, fisiológicas, somáticas, espirituales, que conforman la personalidad de cada cual. Dichas peculiaridades diferentes se denominan estilos. El estilo se relaciona con el conocer, el pensar, el afecto y la conducta.

Kolb, David (1999), describe los estilos de aprendizaje basados en la forma de procesar la información de la siguiente manera:

El estilo divergente: Combina los pasos de aprendizaje de experiencia concreta y observación reflexiva. 
Estilo asimilador: Combina los pasos de aprendizaje de observación reflexiva y conceptualización abstracta.

Estilo convergente: Combina los pasos de aprendizaje de conceptualización abstracta y experimentación activa.

Estilo acomodador: combina los pasos de aprendizaje de experimentación activa y experiencia concreta.

Según Polanco, (1995); Salas, (1995); Tancredi, (1992) \& Abarca (1990), citado por Lobo \& Jiménez (2002), señalan, que se necesita por igual de las cuatro capacidades cognoscitivas: tensión creadora entre el pensamiento abstracto y el concreto, observación reflexiva y la experimentación activa.

\section{Enfoques de enseñanza utilizados por los docentes}

\section{Enfoque transmisivo de enseñanza}

Es en este ámbito donde se enmarca el artículo, al tratar de clarificar y avanzar en el conocimiento de la relación entre los enfoques de enseñanza desde una perspectiva práctica de aula y los Estilos de Aprendizaje siguiendo la terminología, y la descripción propuesta por Alonso y Gallego (1994), citado por Martínez (2007). Donde el principio de individualización es un requerimiento social y por tanto educativo que exige que el trabajo dentro del aula se personalice.

Bautista, Porlán \& Jiménez, (1992), refieren que los docentes deben contemplar como autoexigencia, la coherencia de sus intervenciones con la diversidad de los estudiantes. Desde este enfoque, el proceso de enseñanza-aprendizaje se reduce a transmitir los conocimientos que forman parte de nuestra cultura.

\section{Enfoque constructivista de enseñanza}

Según Coll, Martín \& Mauri, (1993); García (1993), citado por Martínez (2007). El enfoque constructivista, se apoya en los fundamentos pedagógicos socráticos y posteriormente piagetianos, según los cuales, la enseñanza debe actuar sobre el individuo conduciéndole a la transformación o al cambio de su forma de pensar y de sus esquemas de conocimiento. Para que estas modificaciones mentales se verifiquen, la enseñanza debe ajustarse al desarrollo intelectual de los estudiantes a la vez que a sus intereses, capacidades y contexto en que se desenvuelve. 


\section{Relación entre los estilos de aprendizaje y los enfoques de enseñanza}

Un modelo de estilos de aprendizaje clasifica cinco dimensiones de análisis: percepción, entrada, organización, procesamiento y comprensión; pero en una posterior versión suprimen la dimensión de organización -inductivo y deductivo- (Felder, 1998).

Según Freire (1976), para facilitar el aprendizaje en los estudiantes se requiere que la contribución del formador sea mucho más que ser simplemente una fuente de información. Un docente eficiente tendrá que elaborar un programa de formación, facilitar métodos que satisfagan las necesidades específicas de los estudiantes.

\section{Metodología}

El presente estudio se realizó en el recinto universitario URACCAN Las Minas, ubicado geográficamente en Siuna, el cual forma parte de las Regiones Autónomas de la Costa Caribe Nicaragüense -RAAN-. Es un modelo de paradigma cualitativo y se enmarcó en la perspectiva teórica del enfoque etnometodológico.

La población objeto de estudio fueron los discentes y docentes de la carrera Agroforestal en URACCAN Recinto Las Minas, se buscó comprender y analizar los estilos de aprendizaje, asimismo los enfoques de enseñanza que se desarrollan a través de la interacción en los procesos de enseñanza a partir de los resultados, para que en un futuro, sean base de decisiones que permitan incidir en mejorar el quehacer docente y la educación estudiantil.

\section{Resultados}

\section{Estilos de aprendizaje que prevalecen en los discentes}

Se revela que los estilos de aprendizajes en los procesos de enseñanza, corresponden a estilo divergente y acomodador (ver gráfico No. 1). 


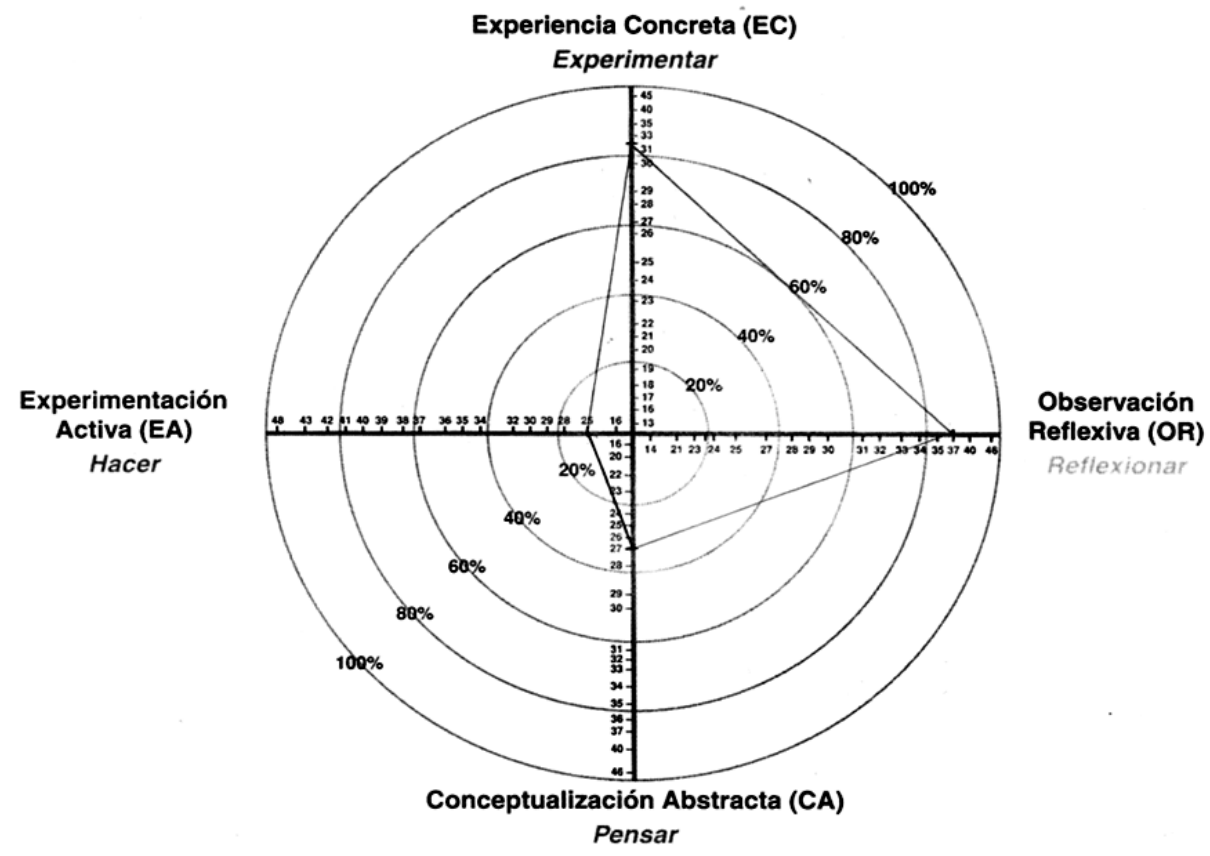

Gráfico No. 1: Diagrama del Estilos de Aprendizaje Divergente.(Fuente: Inventario de estilos en el aprendizaje de Kolb).

En el gráfico se puede observar que el estilo de aprendizaje se encuentra en las cuatro fases, sin embargo, significativamente tiende a inclinarse hacia un estilo divergente combinando los pasos de aprendizaje de experiencia concreta y observación reflexiva. Lo que se fundamenta en lo planteado por Kolb (2000), quien expresa que el estilo de aprendizaje divergente es donde el discente combina los pasos de aprendizaje de experiencia concreta y observación reflexiva y es donde las personas que adoptan este estilo de aprendizaje generalmente observan las situaciones concretas desde diferentes puntos de vista y que en situaciones de aprendizaje formal, tal vez prefiera trabajar en grupos para recopilar información, escuchar con una mente abierta y recibir realimentación personalizada.

Al respecto un estudiante expresa:

Para afianzar mis conocimientos me gusta el trabajo de grupo porque esto nos ayuda a tener ideas y además me permite intercambiar conocimientos, esto porque hay estudiantes que tienen experiencia en determinadas temáticas, que a veces yo no las tengo y viceversa... 
Lo anterior refleja la intencionalidad del discente de interactuar y establecer relaciones para adquirir y construir el conocimiento, lo que se evidencia en un aprendizaje de interacción de pensamiento (Ver gráfico No. 2).

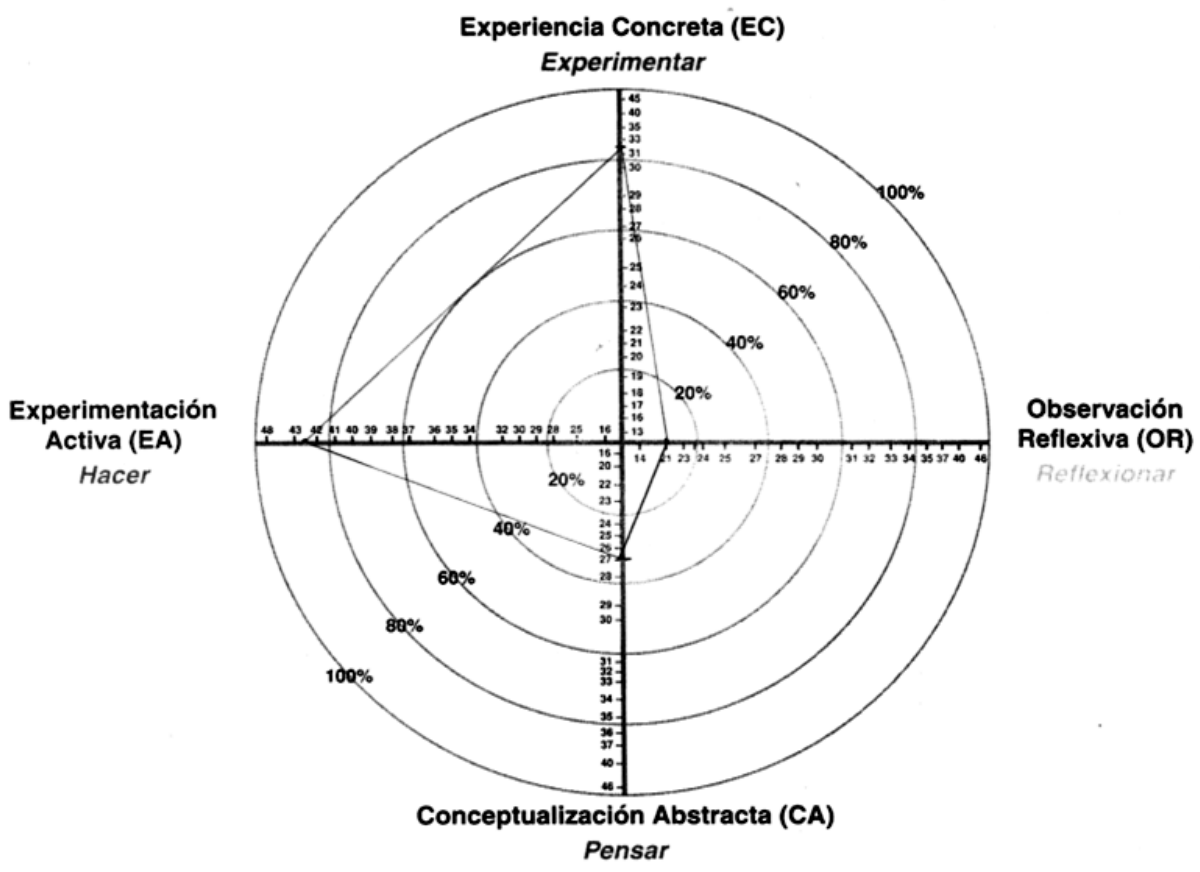

Gráfico No. 2: Diagrama del estilo de aprendizaje acomodador (Fuente: Inventario de estilos de aprendizaje de Kolb).

El diagrama indica que este es un estilo de aprendizaje que significativamente tiende a inclinarse hacia un estilo acomodador combinando los pasos de aprendizaje de la experiencia activa y la experiencia concreta. Este tipo de discente se siente más conforme con lo que él o la docente le proporciona y asume la conducción en el aprendizaje.

Referente a esto un discente expresó:

El aprendizaje lo orienta el docente y este es el que pauta y desarrolla los contenidos que debo como estudiante dominar, porque considero que cada uno de ellos se orienta bajo un programa curricular y de competencia que tengo que dominar.

Lo antes expuesto indica que este tipo de discente le gusta actuar en base a sus sentimientos, así mismo se caracteriza por ser más operativo que propositivo, lo que se traduce en un individuo dependiente del sistema de educación que él emprende. 


\section{Enfoques de enseñanza utilizados por los docentes en el aula de clase}

Los resultados de las entrevistas reflejan que los estilos de aprendizaje de los discentes, se desarrollan en las aulas de clase en torno a dos enfoques de enseñanza: el transmisivo y el constructivista. Las prácticas de estrategias según el enfoque transmisivo, está evidenciado por los objetivos y contenidos de las diversas disciplinas, indicando que los estilos de aprendizaje del discente se convierten en una tarea añadida a la práctica habitual.

La observación realizada en las aulas de clase a los docentes, permitió identificar que la mayoría ejercen una enseñanza con un enfoque transmisivo en un proceso unidireccional docente - discente, cuyo objetivo final es la acumulación de contenidos programático en la mente de los discentes; lo que indica que este proceso está estructurado en torno a una serie de lecciones o temas ordenados lógicamente desde el ámbito disciplinar y que el discente debe asimilar con idéntica estructura.

Por tanto, la acción principal desde este enfoque consiste en la explicación o exposición y conferencia de los contenidos planificados por núcleos temáticos, cuyo objetivo final es la acumulación de contenidos curriculares en la mente de los estudiantes.

Al conversar sobre este punto con una docente de la carrera expresa:

Bueno [...] primeramente se desarrollan los contenidos establecidos en el programa y el plan de clase que corresponde al día de desarrollo de la asignatura y para que los estudiantes fijen el conocimiento se dedican espacios de tiempo a ejercicios de aplicación sobre lo desarrollado, y [...] posteriormente se evalúa ya sea con prueba escrita o exposición para medir el nivel de adquisición y memorización que el estudiante logro captar pero todo sobre lo desarrollado en clase (Entrevista, enero 2009).

El planteamiento anterior se corresponde con la observación realizada en el aula de clase, identificando por tanto, discentes que evidencian un comportamiento pasivo y se convierten en receptores de contenidos producto de un proceso de aprendizaje cognitivo. La enseñanza desde este enfoque se enmarca más bien en un proceso hasta muchas veces monótono en la que sobre salen estrategias de enseñanza que verifican el proceso pasivo del aprendizaje, entre los más frecuentes encontramos la exposición oral, lectura del libro de texto, subrayado esta última, desarrollada por pocos docentes y para desarrollarla se apoyan en la pizarra y en otros medios auxiliares como el uso del data show para reforzar la palabra y mantener la atención.

En este enfoque, el proceso de enseñanza se reduce a transmitir los conocimientos que forman parte de nuestra cultura, así mismo Bautista, Porlán \& Jiménez (1992), señalan que la actividad en el aula se concreta básicamente en explicar los contenidos de una determinada y aislada disciplina, insistiendo en los contenidos conceptuales, 
definiendo correctamente su significado con el argumento lógico que justifica su inclusión en el currículum.

Sin embargo, cuando la enseñanza se desarrolla de acuerdo con el enfoque constructivista, el desarrollo de los estilos de aprendizaje es un trabajo integrado en el quehacer docente, cabe mencionar que este enfoque es practicado por una minoría de docentes del área en estudio. Por otro lado, la enseñanza desde el enfoque constructivista desarrollada por los docentes en las aulas de clase es menos frecuente, y cuando se logra concretar, actúa sobre el individuo, por tanto, el trasfondo del mismo es conducir a la transformación o cambio al discente de su forma de pensar y de sus esquemas de conocimientos.

Referente a ello, en entrevista realizada a un docente expresó: "la enseñanza debe ajustarse al desarrollo intelectual del alumnado -sic- a la vez que a sus intereses, capacidades y contexto en que se desenvuelve".

Por otro lado, este tipo de docente, además de contar con un guión establecido de contenido desde los programas son creativos seleccionando los contenidos en base a problemas de las diversas áreas, teniendo como referente los conocimientos que posee y el estado evolutivo del que aprende. Esto conlleva una secuencia de enseñanza estructurada donde el discente identifica, reconoce y analiza los problemas de aprendizaje, de manera que se da un proceso horizontal donde se comparte y comprende el proceso de aprendizaje.

\section{Relación entre los estilos y enfoques de enseñanza-aprendizaje}

La relación que existe entre los estilos de aprendizaje respecto a los enfoques de enseñanza implementados por el personal docente, se evidencia en dos escenarios que tienen que ver con una relación unidireccional y horizontal docente y discente, de tal manera que se da en un nivel no significativo con respecto a las características de los estilos identificados destacando que ninguno de los docentes entrevistados conoce el estilo de aprendizaje de sus discentes.

En el proceso de enseñanza con un enfoque transmisivo, la mayoría del personal docente, utiliza estrategias de enseñanza tales como: exposición verbal ordenada en base a los contenidos curriculares, los que muchas veces son memorizados tal como se exponen, por consiguiente, el conocimientos se estima cerrado y absoluto, los contenidos se consideran un reflejo exacto de la realidad y se desarrollan en función de acumulación de los mismos.

Por lo consiguiente, se puede decir que existe coherencia con el enfoque de enseñanza y el estilo acomodador, debido a que el personal docente mantiene la planificación establecida en el inicio del curso, existe exigencia a que los trabajos estén bien 
presentados y mantienen un clima de aula ordenado y tranquilo; sin embargo, no hay coincidencia con respecto a este estilo en la cual no se procura poner a trabajar en grupo a discentes de nivel intelectual semejante.

Con base a lo referido anteriormente, una discente expresó:

Tenemos docentes que son muy esquemáticos y que se limitan al desarrollo de contenidos sin tomar en cuenta los puntos de vistas de nosotras, casi no hacemos reflexiones y en esto el docente debería hacer mayor énfasis, porque facilita la retroalimentación y fortalece la discusión.

En cuanto al otro enfoque de enseñanza que implementan los docentes en el área de agroforestería es constructivista en donde se inicia según la observación con la identificación, reconocimiento y análisis de las ideas que los discentes tiene sobre el problema o tarea de aprendizaje. Buscan compartir y comprender el problema o tarea de aprendizaje. Luego identifican el problema y las contradicciones entre lo que dicen y cómo lo utilizan; el docente trabaja con el conjunto de respuestas, la mayoría intuitiva y sustentada en su experiencia, para reconvertirlas en organizadores previos de todo el posterior proceso de construcción cognitiva.

Con respecto al estilo divergente, los docentes que practican el enfoque constructivista y que corresponden a una minoría, expresan y desarrollan características que coinciden con este estilo de aprendizaje entre ellas se visualizó que los docente en el aula de clase tratan de implicar en nuevas actividades a los discentes, les gusta que los mismos jueguen un papel activo tanto en las experiencia como en las relaciones, así como desarrollar el trabajo de equipo hacia una mente abierta, lo que hace que exista una relación entre el estilo de aprendizaje y las estrategias de enseñanza.

Por lo que podemos afirmar que existe una relación directa entre el enfoque constructivista que utilizan los docentes y el estilo de aprendizaje que prevalecen en los discentes objeto de estudio, porque según este enfoque va mas allá de esperar respuestas puntuales y exactas, sino que se basa en reflexiones, experiencias, donde se trata de construir de manera conjunta ese conocimiento y está más claramente marcado con las características adoptadas en el estilo divergente.

\section{Propuesta metodológicas para una enseñanza que fortalezca los estilos de aprendizaje}

Los resultados encontrados en este estudio, dieron elementos significativos para que la docencia oriente sus procesos de enseñanza - aprendizaje en pro de potencializar el conocimiento de los discentes en las aulas de clase. Los que señalan, que no existe una estrecha correspondencia entre los enfoques de enseñanza y los estilos de aprendizaje 
que se ponen en práctica en este proceso. Situación que da paso a proponer algunas orientaciones metodológicas que permitan una mejor conducción del proceso.

Este esfuerzo permitirá articular propuestas que den pautas sobre la relación que debe darse entre docente y discente para ser efectivo y exitosos en el quehacer de la enseñanza y el aprendizaje. Resultados que servirán de base para implementar estrategias didácticas congruentes con los enfoques y estilos de aprendizaje identificados.

\section{Conclusiones}

Los estilos de aprendizaje identificados en la aplicación del instrumento de Kolb corresponden a estilos de aprendizajes: divergente y acomodador, reflejándose con mayor frecuencia el divergente y en segundo lugar el acomodador.

Los Estilos de Aprendizaje se implementan en la carrera agroforestal desde dos enfoques de enseñanza que actualmente coexisten en las aulas: El transmisivo y el constructivista.

La relación que existe entre los estilos de aprendizaje de los discente con respecto a los enfoques de enseñanza implementados por el personal docente, se evidencia en dos escenarios que tienen que ver con una relación unidireccional y horizontal entre docente y discente.

La propuesta está orientada a mejorar el proceso enseñanza - aprendizaje con acciones de vida académica, vinculando los enfoques de enseñanza con los estilos de aprendizaje de los estudiantes de la carrera agroforestal. En ella se plantea desarrollar los Proceso Integrales de Aprendizaje (PIA), en donde el discente es el sujeto del aprendizaje y el docente recrea el conocimiento, y ambos en conjunto establecen una interacción basada en la incorporación de estrategias metodológicas y de estilos de aprendizaje vinculando el principio del pensamiento crítico.

\section{Lista de referencias}

Arríen, J. (2000). Motivación y aprendizaje en el aula de clase. Santillana Madrid.

Askew, Mary. (2000) Cinco modelos de estilos de aprendizaje. Disponible en http:// members.tripod.com/\%20elhogar/\%202000/2000-10/

Bautista, A. Porlán, R. \& Jiménez, A. (1992). Teoría y Práctica del currículo.

Madrid: MEC. 


\section{EDUCACIÓN}

Bawden (1990). Teorías de aprendizaje y estilos de enseñanza. Psicología Evolutiva educativa. Madrid volumen 4.

Brundage y Mackaracher (1980). Estilos de aprendizaje y estrategias de enseñanza para un aprendizaje significativo. Colombia.

Casau, P. (2001). Andragogía. Consultado, el 18 de octubre del 2008. http:/www.galeon. hispavista.com/pcazau/artdid-andra.htm.

De la Torre, S. (1993). Las estrategias metodológicas. Encuentro Maestría Docencia Universitaria, Managua Nicaragua. 2002.

Díaz Barriga, Frida. (1998). Una aportación a la didáctica de la historia. Perfiles Educativos. UNAM.México.

Duriez, M. (2007). Curso de capacitación a tutores "Investigación cualitativa y cuali-cuantitativa".

Fariñas, G. (1995). Motivación y aprendizaje en el aula. Cómo enseñar a pensar.

Felder, R. (1998). Index of Learning Styles. Consultado el 15 de agosto de 2008 en: www.2.ncsu.edu/unity/lockers/users/f/felder/public/ILSdir/ilsweb.html

Freire (1976). Conocimiento para facilitar el aprendizaje en las aula de clase. México.

Gimeno Sancristán, J. (1996). La evaluación en la enseñanza. En Comprender y transformar la práctica. Madrid.

Garfinkel (1967). Instrumentos metodológicos para el estudio de las relaciones interétnicas en el periodo formativo y de consolidación de Estados nacionales. Buenos Aires, CEAL.

Gutiérrez, G. (2003). Módulo de Formulación, Realización y Evaluación de proyectos de investigación. Maestría en métodos de investigación cualitativa, UPOLI.

Judi, A. (1994). Directrices para estudios en base a la técnica de investigación cualitativa.

Knowles, A. (1980). Conocimiento e información como procesos de construcción de aprendizajes. Pontificia Universidad Católica del Perú. Fondo Editorial. Lima.

Kolb, David (1999). Inventarios de estilos de aprendizajes. Educación adulto.Guatemala. Versión 3. 
Leeuwis, Long y Villareal (1991). Conceptos de conocimientos individuales en el quehacer docente. Los estadios en la Psicología. Buenos Aire.

Leichter, (1990). La comprensión del aprendizaje en el aula de clase. PAIDOS.Barcelona.

López, G. (2007). Planificación del estudio. Métodos y técnicas de aprendizaje. [En línea]. ECEM. Disponible: ww.ecem.mil.bo/documentos/tecnicas\%20de\%2oestudio. pps -[ 2008, 9 abril].

Lobo, N. y Jiménez, F. (2002). Los estilos de aprendizaje y el desgranamiento

universitario en carreras de informática. Primeras Jornadas de Educación en Informática y TICS en Argentina.

Márquez Vázquez, F. (1999). El aprendizaje estratégico. Aula XXI. Santillana. España.

Martínez, P. (2007). Orientaciones didácticas para trabajar los estilos de Aprendizaje en el aula (Educación Secundaria). En II Congreso Internacional de Estilos de Aprendizaje. Universidad de Concepción-Chile.

Marreros, M. (2007). Estilos de aprendizaje y su impacto en el proceso enseñanza aprendizaje. Aplicación de terapias ocupacional en difusión. Departamento de Terapia Ocupacional.

Morrish, I. (1978). Cambio e innovación en la enseñanza. Salamanca, Anaya-2.

Poon, James. (2000), Entendimiento de los estilos de aprendizaje los estudiantes: Implicaciones para la enseñanza.. "La Revista Internacional de Sociología y Política Social; Patrington.Vol.20, Iss 11/12.

Piaget, Jean. (1994). La teoría del constructivismo de Piaget. [En Linea]. Disponible en: Ibe. unesco.org

Pozo, J. (1999) El Aprendizaje Estratégico. España.

Revilla, D. (1998). Estilos de aprendizaje, Temas de Educación, Segundo

Seminario Virtual del Dep. de Educación de la Pontificia Universidad Católica del Perú, disponible [en línea]. http://www.pucp.edu.pe/ temas/estilos.html.

Rendón, M. (1999). El desarrollo cognoscitivo y su relación con el aprendizaje.

Rogers y Taylor (1998). Los estilos de aprendizajes en psicología y educación. 
Salas, R. (1995). Aprendiendo y diseñando con estilo. Edición universitaria Austral de Chile, Dirección de Investigación y Desarrollo.

Silverman, L. (1988). Estrategias de aprendizaje. Experiencias creadas desde el aula, Chile 5 ta edición.

Tancredi, B. (1992). Procesamiento de información y rendimiento en matemática: un estudio a partir de los estilos de aprendizajes de los estudiantes. En informe de investigación educativas. Vol. VI, Nros.1 y 2.

Taylor y R. Bogdan (1996). Introducción a los métodos cualitativos de

investigación. Paidos.

Taylor y Beniest, (2006). Enseñanza y aprendizaje desde una perspectiva de conocimiento significativo.

Teber, S. (2003), El perfil del profesor mediador. Santillana, España.

Torres, S. (2000). Quiero aprender... [en línea]. Bogota: Universidad Nacional de Colombia. Disponible: is.unal.edu.co/profesores/lucas/escritos/QAprende. pdf - [2008, 5 marzo]

Tunnermann, C. (2000). Retos y perspectivas de la educación superior.

Revista de la Universidad Centroamericana. Encuentro No. (55), 101- 106.

Zabala, A. (1995). La práctica educativa. Cómo enseñar. Barcelona: Grao. 